\title{
Metallic laser clad coatings: on the processing-microstructure-property relationships
}

\author{
V. Ocelík, U. de Oliveira \& J. Th. M. De Hosson \\ Department of Applied Physics, Materials innovation Institute, \\ University of Groningen, The Netherlands
}

\begin{abstract}
A thick metallic coating that is resistant against high loading impact, severe wear and corrosion at high temperatures can be produced through the laser clad method. This work introduces the Orientation Imagining Microscopy based on electron backscatter diffraction in a scanning electron microscope as a very powerful instrument for studying relationships between processing parameters and the microstructure of individual laser tracks and final coatings formed by overlap. The study has been performed on thick $(\sim 1 \mathrm{~mm})$ Co-based coatings prepared by a $2 \mathrm{~kW} \mathrm{CW} \mathrm{Nd:YAG} \mathrm{laser} \mathrm{cladding} \mathrm{on} 42 \mathrm{CrMo} 4$ steel substrate using substantially different laser beam scanning speeds.

OIM provides new insights into the microstructure of laser clad coatings and yields very useful information concerning the directional grow of individual grains, the solidification texture and the shape of solidification front during laser cladding. Strong correlations between these parameters and laser cladding speed as well as the presence of internal interfaces with the sharp microstructural and mechanical properties changes are presented and discussed.
\end{abstract}

Keywords: laser cladding, microstructure, orientation imaging microscopy.

\section{Introduction}

Laser cladding is an advanced hard facing technology that can deposit a hard layer with a controlled thickness onto the selected area of a metallic substrate. It has a low energy input and causes less distortion of the component as compared 
to conventional welding base processes. A detailed description of laser cladding process and its characteristics has been presented in the literature [1-3].

Processing windows have been studied intensively during last two decades to define technological regimes that produced thick and defect free coatings with a required thickness. It was found, that the laser cladding process is technologically a quite robust procedure. Laser power $P$, laser beam scanning speed $S$ and powder feeding rate $F$ (or combinations thereof) are the main technological parameters that control the laser cladding with the powder blowing technique [4-6]. Rapid solidification in the surface layer is the characteristic feature for the laser cladding process and in some conditions the coatings formed by cooling rates higher than $10^{3} \mathrm{~K} / \mathrm{s}$ show very fine, non-equilibrium or even amorphous microstructures. Moreover, the recently developed fiber laser with a nominal power exceeded $5-10 \mathrm{~kW}$ provides a possibility to form laser clad coatings at even higher processing speeds and cooling rates [7, 8].

However, the relations between the processing parameters and the final microstructure and properties of laser clad coatings were studied only scarcely $[9,10]$ and it was mainly focused to the relation between processing speed, secondary dendrite spacing, hardness and wear resistance of Co-based alloys.

The modern Orientation Imagining Microscopy (OIM) technique [11] which combines advantages of Scanning Electron Microscopy (SEM) observations with the information about crystal/grain orientations typical for transmission electron microscopy (TEM) observation may be the very efficient method to study complicated microstructures formed during the laser cladding and fast solidification associated to it. This work is the first attempt to study systematically microstructure of the individual laser tracks and laser clad coatings using OIM.

The Eutroloy 16012 powder was selected for this work because of wide use of Stellite type Co-based alloys in laser coatings applications for hardfacing, wear and corrosion resistance at elevated temperatures. These coatings are extensively used for hardfacing valves, shafts and many other industrial components [3]. The wear resistance of this type alloys and their laser clad coatings is attributed to the high hardness of chromium rich $\mathrm{M}_{7} \mathrm{C}_{3}$ carbides [12] and to the formation of an oxidation protective layer at high temperatures [6]. The microstructure of rapidly cooled Stellite type alloys with carbon content lower than $\sim 2 \mathrm{wt} \%$ is simply characterized as hypoeutectic structure containing face-centered cubic (fcc) Co rich primary dendrites ( $\alpha$-cobalt based solid solution) surrounded by an interdendritic eutectic with $\mathrm{W}, \mathrm{Cr}$ and Co carbides. It results in a relatively strong electron back scattering diffraction (EBSD) signal originated from $\alpha$-Co dendrites and interdendritic eutectic component [13]. Due to the coherence between the fcc phase in primary dendrites and in eutectic, the fine dendritic microstructure is not manifested on low magnification OIM scans.

\section{Experimental}

A $2 \mathrm{~kW} \mathrm{Nd:YAG} \mathrm{Rofin} \mathrm{Sinar} \mathrm{CW20} \mathrm{high} \mathrm{power} \mathrm{laser} \mathrm{source} \mathrm{was} \mathrm{used} \mathrm{to}$ prepare all single laser tracks or continuous coatings. The laser beam with 
wavelength of $1.06 \mu \mathrm{m}$ was focused $16 \mathrm{~mm}$ above the substrate surface forming the circular beam spot size of $3 \mathrm{~mm}$ in diameter with a Gaussian distribution of energy density inside the beam on the treated surface. Side powder cladding setup [6] was used in which the powder is delivered by carrier gas (Argon) from a side against the cladding hill using the commercial powder nozzle (IWS Dresden) mounted under angle $35^{\circ}$ to the surface normal. Four different laser beam scanning speeds $S=1,2.5,7.5$ and $15 \mathrm{~mm} / \mathrm{s}$ were used to vary the solidification rate. Different laser power and powder feeding rates from intervals $600-1750 \mathrm{~W}$ and $21-250 \mathrm{mg} / \mathrm{s}$, respectively, were used to achieve the laser tracks with a similar height and width for all four different scanning speeds.

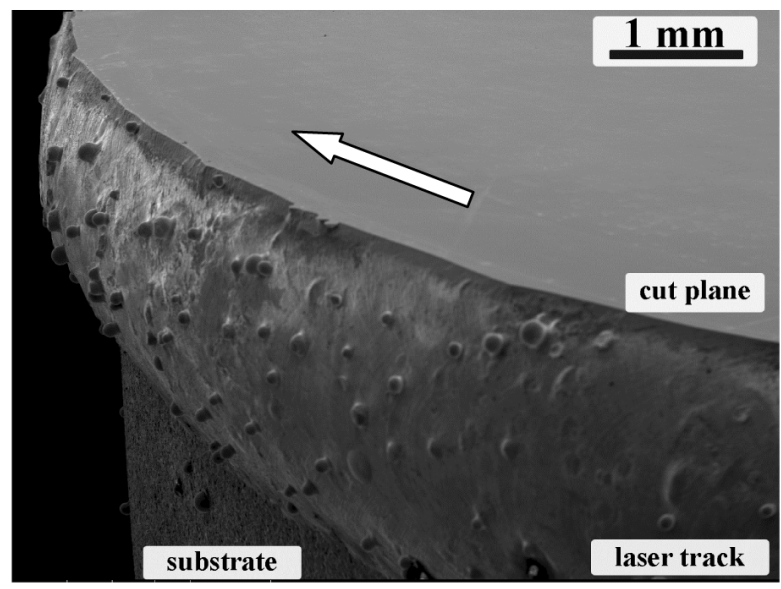

Figure 1: $\quad$ SEM image of laser clad sample cut in direction perpendicular to substrate bar axes and almost parallel to cladding direction. The arrow indicates direction of substrate movement during laser cladding.

All laser clads were deposited on the substrate consisting of $42 \mathrm{CrMo} 4$ steel bar with diameter of $25 \mathrm{~mm}$. The bar was horizontally mounted into a rotation clamp to rotate bar around its axis. The rotation speed at the bar surface actually represents the laser beam scanning speed during the laser cladding. At the same time the substrate bar was slowly moving in the direction of bar axis, to create the laser track in a form of coil at the bar surface. During the clad of single laser tracks this axial movement was set to perform a displacement of one laser track width after each single rotation. To create the laser coatings this displacement has been set to $2 / 3$ of the single laser track width after each rotation to form coatings with $33 \%$ laser tracks overlap. The advantage of such arrangement is that the cutting of the bar in an appropriate plane perpendicular to the bar axis allows to observe the microstructure in planes almost parallel to the cladding direction, slowly moving from one to the other side of laser track. Figure 1 demonstrates this on the sample with a single laser track. Samples cut surfaces were subjected to the standard metallographic procedure that started with 
grinding on grind papers $(180,300,500,800$ and 1200), continued with polishing in diamond particles suspensions $(9,6$ and $3 \mu \mathrm{m}$ size) and finished with a long time ( $\sim 2$ hours) polishing in Struers OP-U suspension. This procedure results in an excellent EBSD signal coming from the laser clad area as Kikuchi patterns easily indexed by fcc Co crystal data. TSL back scatter diffraction system installed inside Philips XL30 FEG scanning electron microscope was used to collect OIM data. The samples were mounted in the scanning electron microscope so that the sample normal is at 76 degree with respect to the electron beam direction. The diffraction image formed on the phosphorous screen by diffracted backscattered electrons is digitized and transmitted to the computer. The orientation of the crystal lattice at each predetermined sample surface point is then automatically determined in coordinate system connected with the sample. The arrays of indexed points used in the present experiments were hexagonal networks with $\mathrm{y}$ and $\mathrm{x}$ increments equal to $3 \mu \mathrm{m}$. Between $260 \mathrm{k}$ and $520 \mathrm{k}$ lattice orientations were determined in each observation using automated indexing regime [14].

\section{Results and discussion}

\subsection{Grains structure}

Figure 2 shows the typical OIM image collected from Eutrolloy 16012 single laser track on its transversal cross-section. This Image Quality (IQ) map consists from about 520k points.

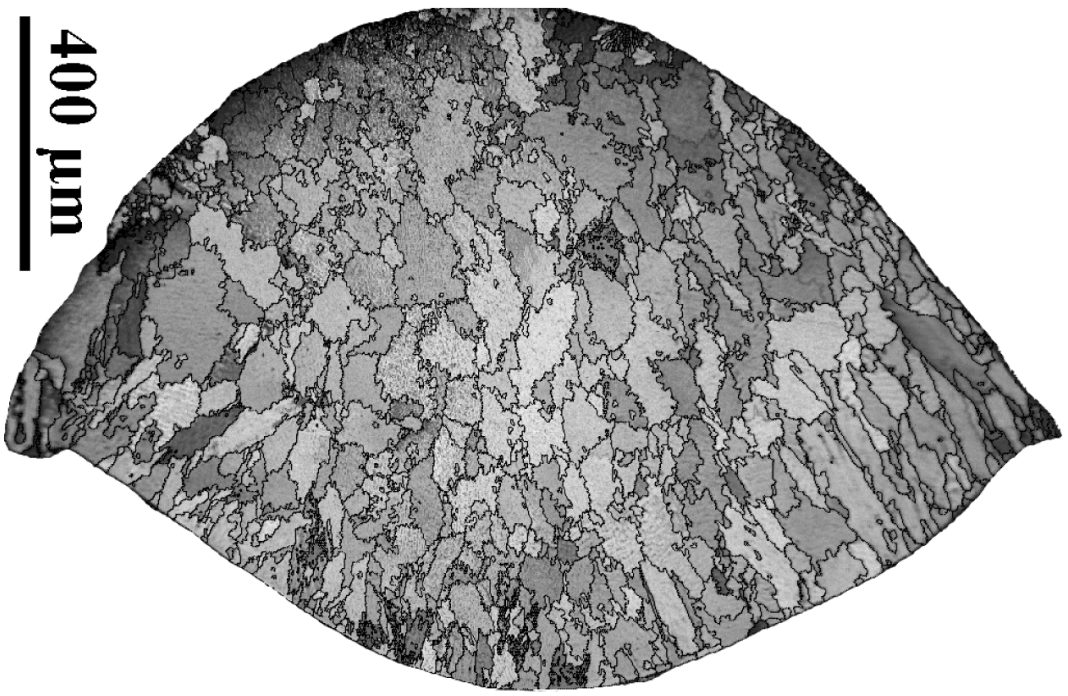

Figure 2: Typical OIM image quality map with grain boundaries on perpendicular cut of single laser track made at laser beam scanning speed $1 \mathrm{~mm} / \mathrm{s}$. 
The grey intensity of individual points corresponds to IQ parameter that reflects the quality of Kikuchi pattern for that particular point and number of detectable Kikuchi lines in it. In some grains one could recognize slight variations in grey intensity due to the presence of small dendrites (size $\sim 4 \mu \mathrm{m}$ ) in the microstructure that results in higher quality Kikuchi pattern than interdendritic space between them. Grain boundary lines presented in Fig. 2 were constructed electronically using the condition that the grain boundary between two scanned points exists when the crystal misorientation angle between these two points exceeds 5 degrees. This figure demonstrates few advantages of OIM technique in comparison with standard SEM observations. The size and shape of the grains could be easily detected and statistically investigated due their digital character of OIM maps. The average grain size diameter calculated from data in Fig. 2 is $88 \mu \mathrm{m}$. The grain diameter size is spread from 20 to $180 \mu \mathrm{m}$ and grains are or equiaxial or only slightly elongated in directions pointing from their bottoms to a point at the top of laser track hill.

However, a completely different picture appears when the longitudinal cut in the middle of the laser track shown in Fig. 2 is observed as Fig. 3 clearly demonstrates.

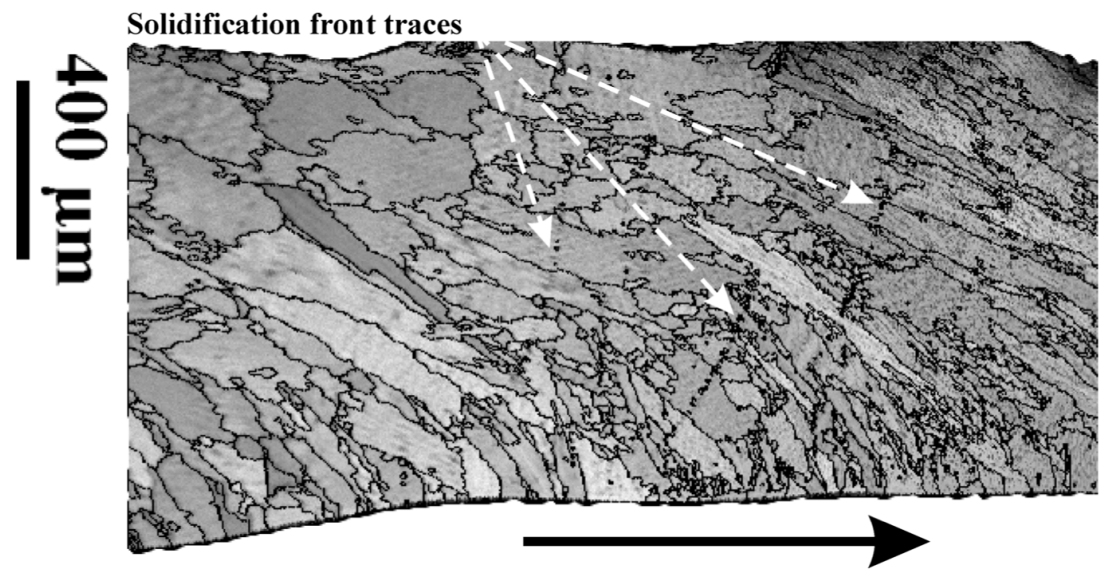

Figure 3: $\quad$ OIM image quality map with grain boundaries on transversal cut in the middle of single laser track made at laser beam scanning speed $1 \mathrm{~mm} / \mathrm{s}$. Black arrow indicates the substrate movement direction during cladding.

The average grain shape aspect ratio is about 0.4 , much smaller than on transversal cut. Moreover, the IQ map in Fig. 3 clearly shows the traces of solidification front in the form of group of small black objects on parabolic lines that witness low qualities of EBSD patterns. This is the manifestation of impurities and oxides segregation at such a low laser cladding speed that results in marking of the solidification front shape in a particular moment of laser cladding process. Majority of the grains in the central and lower part of the laser 
track are strongly elongated in the direction locally perpendicular to the shape of solidification front. It is inherent to expect, that these directions of grains elongation correspond to the direction of their growing, because the main heat transfer during solidification is realized in direction perpendicular to the solidification front. We may conclude here that the grain size and their shape information from only transversal cut observation are not satisfactory for description of the microstructure formed during laser cladding process. More complete picture is offered by observation of longitudinal cross section.

\subsection{Influence of laser beam scanning speed}

Figure 4 summarizes the grain size and grain shape observations from transversal and longitudinal cross-sections for all four laser beam scanning speeds.

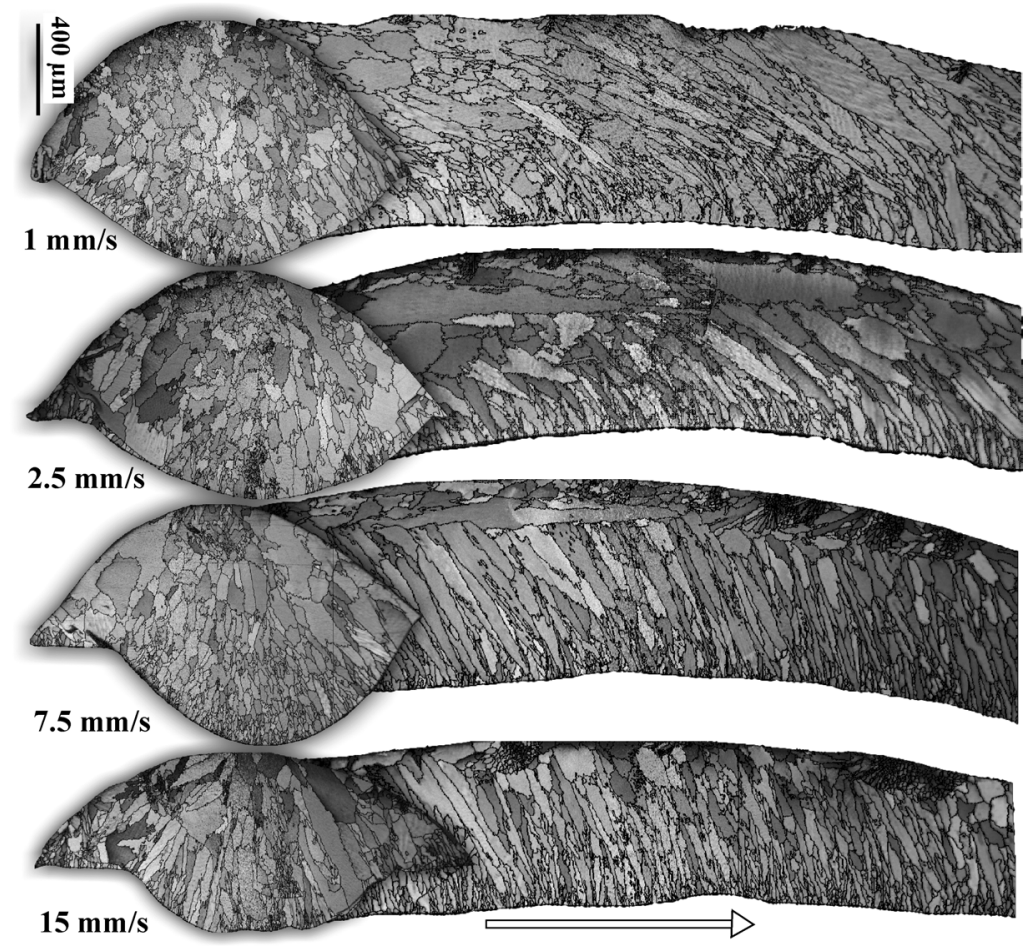

Figure 4: Image quality figures with grain boundaries from transversal (left) and longitudinal (right) cuts of single laser tracks formed at four different laser cladding speeds.

Transversal cross sections show a gradual increase of number of elongated grains with increasing laser beam scanning speed. However, this increase is not induced by a higher presence of elongated grains in the microstructure but it is a simple consequence of gradual change of the average angle between the long 
axis of elongated grains and the cladding direction, represented in Fig. 4 by an arrow. To collect quantitative data how this angle changes with the cladding speed we performed the following procedure. First, each detected grain in the longitudinal microstructures from Fig. 4 was replaced by an ellipse that fits this grain shape. In the second step only those grains that shown corresponding ellipse shape ratio (ratio between short and long ellipse axis) lower than 0.3 were separated from the microstructure. Finally, the average angle between the long axes of ellipses represented these grains and cladding direction was calculated for each cladding speed together with the average grains shape ratio. This procedure is explained in the insert of Fig. 5 where an example of the selection of elongated grains is shown for microstructure formed at $15 \mathrm{~mm} / \mathrm{s}$ scanning speed. Corresponding ellipses, which fit individual elongated grains, are also visible. There is also one example of an angle between the long axis of one grain and the cladding direction shown. The average values were calculated as a statistical mean value from all elongated grains for each scanning speed separately.

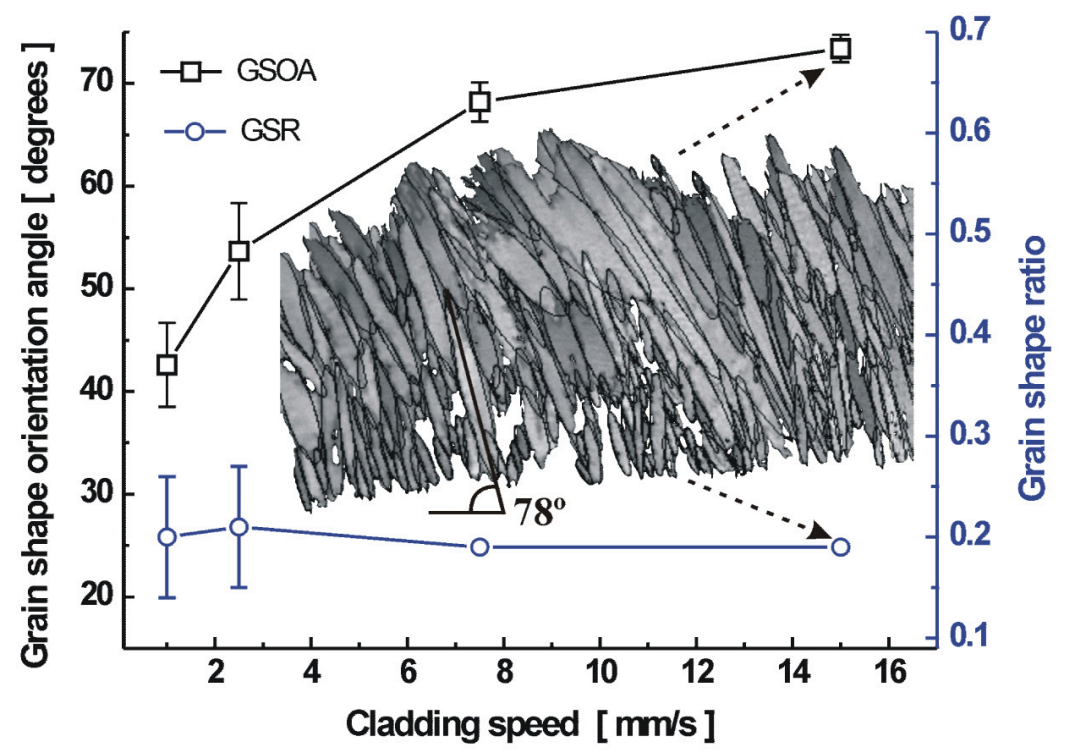

Figure 5: Example of selected elongated grains from longitudinal cut at 15 $\mathrm{mm} / \mathrm{s}$ cladding speed (insert) and results of statistical study of grains orientation angle and grains shape ration as a function of cladding speed.

Graphs in Fig. 5 show the dependence of average grain shape orientation angle and grain shape ratio on the laser beam cladding speed. While the grain shape ratio seems to be not dependent on the scanning speed; keeping its value around 0.2 for all scanning speeds, the average grain shape orientation angle 
substantially increases from $43^{\circ}$ to $74^{\circ}$ with increasing cladding speed. At the same time the variation of this average angle decreases substantially from $10 \%$ at cladding speed of $1 \mathrm{~mm} / \mathrm{s}$ to $2 \%$ at cladding speed of $15 \mathrm{~mm} / \mathrm{s}$.

\subsection{Solidification texture}

The OIM data contains information about the crystal orientation for each point of SEM scanned surface. Therefore it is easy not only to construct grain boundaries but also to study texture in observed microstructures [15]. However, EBSD signal has to be collected from large number of grains. The presence of strong texture inside the laser clad Co based coatings was already concluded by Olivera et al. [16] when measuring the internal microstrains by diffraction of synchrotron radiation. To prove the hypothesis that grains are elongated due to their growth in a direction perpendicular to the solidification front we studied the texture of microstructures formed by different laser beam velocity. Our OIM observations on longitudinal cross-section confirmed the strong solidification texture for all four laser beam scanning speeds. It is the fiber type of texture, when one $<100>$ direction in fcc cubic crystal has a preferable orientation in a coordinate system fixed to the sample whereas others two $<100>$ directions are free. This type of texture often results from directional solidification that prefers the crystal grow in [100] direction for fcc structures [17].
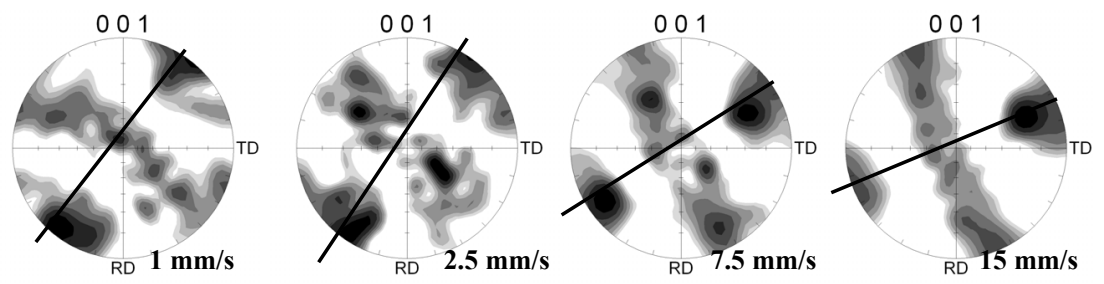

Figure 6: The fibre solidification texture detected by OIM observations on longitudinal cross-sections strongly corresponds to the grain orientation angle.

The Figure 6 demonstrates this situation in the form of (001) pole figures. There is a strong tendency for clustering in one direction while two others form a band crossing the pole figure in its centre. Rolling Direction (RD) in these pole figures represents actually the cladding direction of our samples. It is not surprising that angles measured between the preferable orientation and RD direction correspond to the grain shape orientation angles presented in Fig.5 for all laser beam scanning speeds. This confirms that grains on longitudinal crosssection are elongated in [001] direction and this direction tends to be perpendicular to the shape of solidification front. However, the OIM texture study performed on the transversal cross-sections [13] did not confirm such a strong texture as shown in Fig.6. Simple explanation is hidden in 3D dimensional character of the solidification front shape. 


\subsection{Internal interfaces due to the laser tracks overlap}

Here we present OIM observations performed on coatings prepared by overlap of individual laser tracks. Figure 7 shows at the top OIM image quality map and grain boundaries on the transversal cut of coating prepared at laser beam scanning speed of $15 \mathrm{~mm} / \mathrm{s}$. An overlap of 5 individual laser tracks may be recognized on this transversal cross-section. The longitudinal cross-sections made at different planes are shown on the bottom part of Fig. 7.

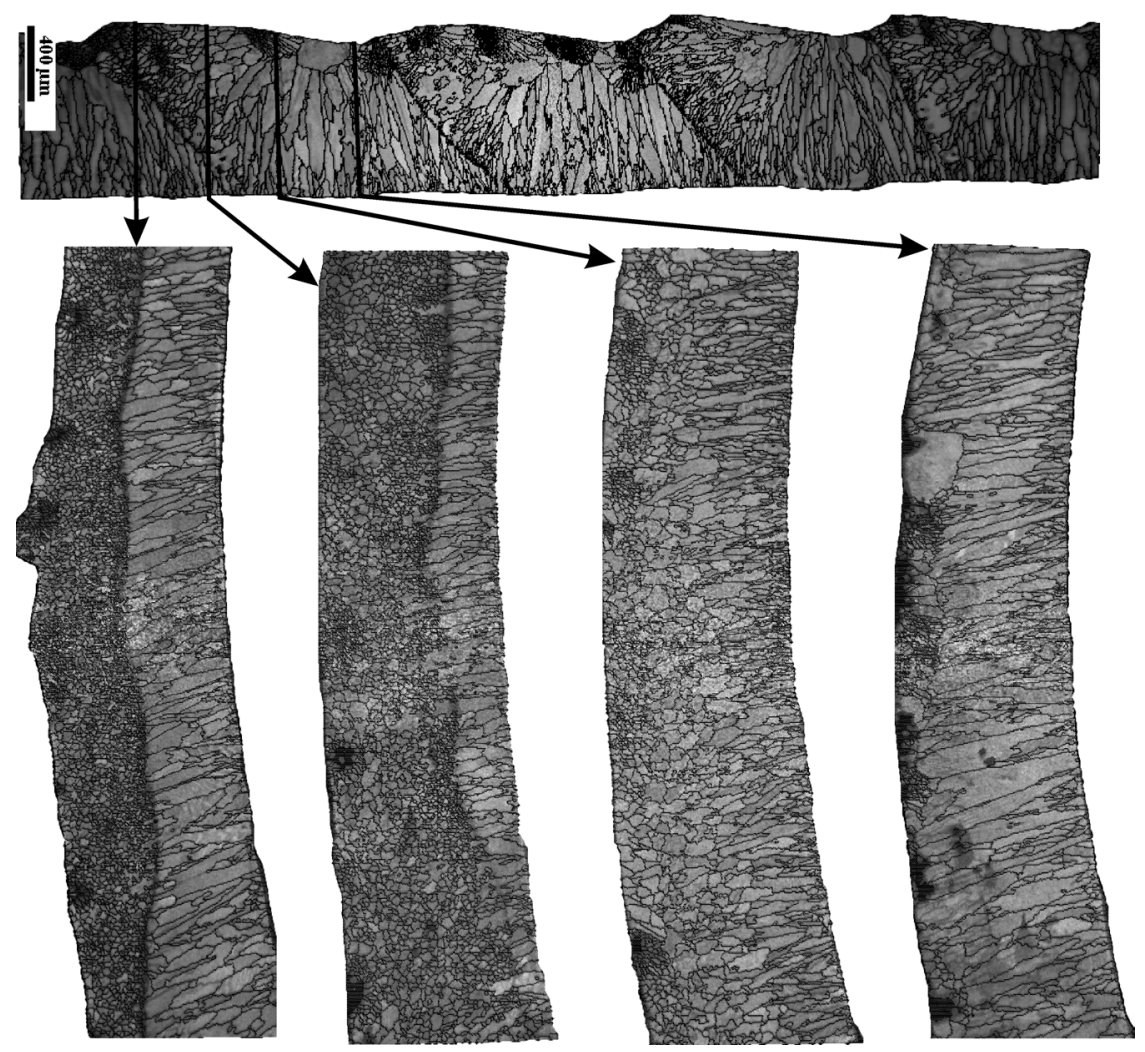

Figure 7: OIM image quality maps with grain boundaries on transversal (up) and 4 longitudinal (bottom) cuts performed at marked positions trough laser clad coating.

Two longitudinal cuts that intersect track overlap boundary show a very sharp change of characteristic grain size directly on this boundary. The presence of such microstructural boundary inside the coating could be less desirable from the point of view of the local sharp change of functional properties of the coating [16]. A comparison of different laser cladding shows that such a sharp microstructural interface is most pronounced in the coating prepared with the highest laser beam scanning speed, e.g. $15 \mathrm{~mm} / \mathrm{s}$. 


\subsection{Microhardness profiles}

Figure 7 summarizes the vertical Vickers' hardness profiles measured with indenter load of $4 \mathrm{~N}$ on single laser tracks and coatings prepared with all laser beam scanning speeds. It is interesting to note, that for the single laser tracks made at higher scanning speeds $(7.5$ and $15 \mathrm{~mm} / \mathrm{s})$ the hardness of the substrate at Heat Affected Zone (HAZ) is substantially higher than the hardness of the coating itself. This is consequence of hardening ability of the substrate steel material. Higher cooling rates associated with faster scanning speeds lead to a higher amount of martensite resulting in higher hardness inside HAZ. The laser beam scanning speed influences the hardness inside laser tracks only slightly. Hardness of coatings is substantially higher than hardness of individual laser tracks made with the same laser beam scanning speeds that is probably raised by precipitation hardening presented during thermal cycling when laser tracks overlap occurs. The same thermal treatment leads to the substantial decrease of hardness inside HAZ, where a decomposition of martensite occurs.
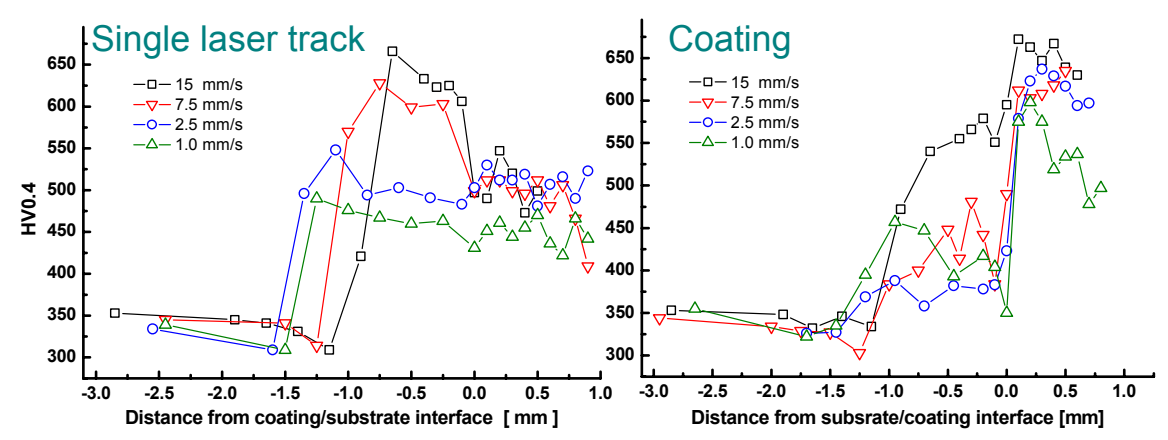

Figure 8: Vickers' microhardness profiles measured on single laser tracks (left) and coatings prepared by different laser beam scanning speeds.

\section{Conclusions}

Orientation Imaging Microscopy provides new insights into the microstructure and texture of thick metallic laser clad tracks and coatings. Cobalt based laser tracks prepared by different laser beam scanning speeds contains relatively large, strongly elongated grains with a strong solidification fibre texture. The direction of this texture together with grain shape orientation strongly depends on cooling rate, e.g. laser beam processing speed.

Conclusions about microstructural features that are based only on a transversal cut of laser track may be misleading. The longitudinal cross-section at the centre of the laser track provides much more information concerning the grain size, the grain shape and the solidification texture of the microstructure formed during laser cladding. 
The possibility for a direct observation of the shape of solidification front at low laser beam scanning speeds has been discovered due to the sensitivity of OIM technique towards impurity phases segregated at the solid/liquid interface.

Formation of thick Co-based coatings via the overlap of individual laser tracks introduces sharp microstructural discontinuities that are more pronounced in coatings made at higher speeds. The laser track overlap may also substantially change the functional properties not only inside the coatings but also inside the heat affected zone.

\section{Acknowledgement}

The work is part of the research program of M2i (Materials Innovation Institute, The Netherlands).

\section{References}

[1] Steen W.M., Laser Materials Processing, 3-rd edition, Springer-Verlag London Limited: London, 408p, 2003.

[2] Toyserkani E., Khajepour A. \& Corbin S., Laser cladding, CRC Press: London, 260p, 2005.

[3] Ion J.C., Laser Processing of Engineering Materials, Elsevier ButterworthHeinemann: Amsterdam, 556p, 2005.

[4] Yellup J.M., Laser cladding using the powder blowing technique. Surface and Coatings Technology, 71(2), pp. 121-128, 1995.

[5] Oliveira de U., Ocelík V. \& De Hosson J.Th.M., Analysis of coaxial laser cladding processing conditions. Surface \& Coatings Technology, 197(2-3), pp. 127-136, 2005.

[6] Ocelík V., Oliveira de U., de Boer M. \& De Hosson J. Th. M., Thick Cobased coating on cast iron by side laser cladding: Analysis of processing conditions and coating properties. Surface \& Coatings Technology, 201(12), pp. 5875-5883, 2007.

[7] Nowotny S., Scharek S. \& Schmidt A., Advanced laser technology applied to cladding and buildup. Welding Journal, 86(5), pp. 48-51, 2007.

[8] Partes K., Seefeld T., Sepold G. \& Vollertsen F., High efficiency laser cladding at elevated processing speed. Proc. Of the ICALEO 2005 conference on Laser Materials Processing, Eds. A. Ostendorf, A. Hoult \& Y.F. Lu, Miami (FL), USA, October 31 - November 3, pp. 621-627, 2005.

[9] Frenk A., Henchoz N. \& Kurz W., Laser cladding of a cobalt-based alloy: Processing parameters and microstructure. Z. Metallkd. 84(12), pp. 886892, 1993.

[10] Frenk A. \& Kurz W., Microstructural effects on the sliding wear resistance of a cobalt-based alloy. Wear, 174(1-2), pp. 81-91, 1994.

[11] Dingley D.J., The development of automated diffraction in scanning and transmission electron microscopy (Chapter 1). Electron Backscattered diffraction in Materials Science, ed. A.J. Schwartz, M. Kumar \& B.L. Adams, Kluwer Academic/Plenum Publishers: New York, pp. 1-16, 2000. 
[12] Davis J.R., ASM Handbook. Properties and Selections: Nonferrous Alloys and Special-Purpose Materials, ASM International: Material Park, $\mathrm{OH}$, Vol.2, 446p, 1990.

[13] Oliveira de U., Laser treatment of alloys: processing, microstructure and structural properties, PhD Thesis, University of Groningen, 128p, 2007.

[14] Wright S.I., Fied D.P. \& Dingley D.J., Advanced software capabilities for automated EBSD (Chapter 13). Electron Backscattered diffraction in Materials Science, ed. A.J. Schwartz, M. Kumar \& B.L. Adams, Kluwer Academic/Plenum Publishers: New York, pp. 141-152, 2000.

[15] Rajan K., ibid, (Chapter 3), pp. 31-38.

[16] Oliveira de U., Ocelík V. \& De Hosson J.Th.M., Microstresses and microstructure in thick cobalt-based laser deposited coatings. Surface \& Coatings Technology, 201(14), pp. 6363-6371, 2007.

[17] Kurz W. \& Fisher D.J., Fundamentals of Solidification, Trans Tech Publications Ltd: Switzerland, 245p, 1986. 